\title{
Laparoscopic repair of hiatal hernia
}

\author{
Ju Sik Yun ${ }^{1}$, Kook Joo $\mathrm{Na}^{1}$, Sang Yun Song ${ }^{1}$, Seok Kim ${ }^{1}$, Eunchong Kim², In Seok Jeong ${ }^{2}$, Sang Gi Oh${ }^{2}$ \\ ${ }^{1}$ Lung and Esophageal Cancer Clinic, Chonnam National University Hwasun Hospital, Chonnam National University School of Medicine, \\ Jeollanam-do, South Korea; ${ }^{2}$ Department of Thoracic and Cardiovascular Surgery, Chonnam National University Hospital, Chonnam National \\ University School of Medicine, Gwang-ju, South Korea \\ Contributions: (I) Conception and design: JS Yun, SY Song, KJ Na; (II) Administrative support: S Kim, IS Jeong, SG Oh, Eunchong Kim; (III) \\ Provision of study materials or patients: JS Yun, IS Jeong, SG Oh; (IV) Collection and assembly of data: JS Yun, S Kim, KJ Na; (V) Data analysis and \\ interpretation: JS Yun, SY Song, E Kim; (VI) Manuscript writing: All authors; (VII) Final approval of manuscript: All authors. \\ Correspondence to: Kook Joo Na, MD, PhD. Lung and Esophageal Cancer Clinic, Department of Thoracic and Cardiovascular Surgery, Chonnam \\ National University Hwasun Hospital, 322 Seoyang-ro, Ilsim-ri, Hwasun-eup, Hwasun-gun, Jeollanamdo 58128, South Korea. \\ Email: kjna1125@hanmail.net.
}

Background: Laparoscopic hiatal hernia repair is a complex surgery typically performed by general abdominal surgeons because it typically involves an abdominal approach. Here, we report our experiences on laparoscopic repair of hiatal hernia as thoracic surgeons.

Methods: Based on our experience of minimally invasive esophageal surgery (MIES) for esophageal cancer, we began performing laparoscopic repair of hiatal hernia in 2009. We analyzed the surgery-related data and postoperative outcomes of 18 consecutive patients we operated on from 2009 to 2017.

Results: There were 1 male and 17 female patients with a median age of 73 years (range, 37-81 years). Ten of 14 symptomatic patients experienced reflux symptoms, such as heartburn. Four patients had a history of prior abdominal surgery. Hiatal hernia types I, II, III, and IV were observed in 3, 9, 5, and 1 patients, respectively. Two (11.1\%) laparoscopic procedures required conversion. Modified Collis gastroplasty was used as an esophageal lengthening procedure in 5 patients (27.8\%). Mean operation time was $213.8 \pm 70.1$ minutes and mean hospital stay was $6.2 \pm 1.5$ days. There were no postoperative complications. At the last follow-up, 15 patients $(83.3 \%)$ were asymptomatic; however, 3 (16.7\%) complained of reflux or dysphagia. Recurrent hiatal hernia was detected on an esophagogram in only 1 patient at 3.5 years after laparoscopic surgery.

Conclusions: Laparoscopic repair of hiatal hernia is a feasible technique with a satisfactory surgical outcome. Importantly, it can be performed by thoracic surgeons who are experienced in the laparoscopic approach.

Keywords: Hernia; laparoscopy; surgery

Submitted Apr 02, 2019. Accepted for publication Aug 12, 2019.

doi: $10.21037 /$ jtd.2019.08.94

View this article at: http://dx.doi.org/10.21037/jtd.2019.08.94

\section{Introduction}

It is difficult to accurately identify the incidence of hiatal hernia, as asymptomatic hiatal hernia often goes undetected. However, symptomatic hernia should be examined in association with gastro-esophageal reflux disease (GERD) in terms of its pathophysiology, as the incidence of GERD is on the rise worldwide $(1,2)$. Indeed, the incidence of GERD is lower in the east compared to that in the west; nonetheless, based on diagnosis rate, its incidence is increasing in our country (3). Although medical treatment, including proton pump inhibitors, is the preferred treatment for controlling symptoms, surgical interventions may be needed, depending on the severity of symptoms and type of hernia involved $(4,5)$. Recently, laparoscopic repair has been widely performed because it has multiple benefits. It is generally performed by a general abdominal surgeon because it usually involves an abdominal approach. 
However, it is also performed by thoracic surgeons who have accumulated relevant experiences in laparoscopic surgery, and successful surgical outcomes have been reported (6). Here, as thoracic surgeons with experience in minimally invasive esophageal surgery (MIES: thoracoscopic esophagectomy + laparoscopic gastric mobilization) for esophageal cancer, we share our experiences in laparoscopic repair of hiatal hernia and analyze our surgical outcomes.

\section{Methods}

\section{Patients}

The present study protocol was reviewed and approved by the Institutional Review Board of Chonnam National University Hwasun Hospital, which waived the requirement for informed patient consent based on the retrospective nature of the work (approval No. CNUHH-2019-056). We have been performing MIES for esophageal cancer since late 2004 with an average of more than 30 cases every year. A single surgeon performs MIES using thoracoscopy and laparoscopy. We have previously reported that MIES is a complex surgery with a substantial learning curve (7); based on the experiences in such laparoscopic surgery, we began performing laparoscopic repair of hiatal hernia in 2009. Eighteen patients with primary hiatal hernia who underwent the laparoscopic surgery from 2009 to 2017 were examined; four patients who developed secondary hiatal hernia after undergoing esophageal cancer surgery were excluded.

Routine preoperative tests were performed (e.g., physical examination, standard laboratory tests, and pulmonary function tests); additionally, esophagogastroduodenoscopy, chest and abdominal computed tomography, and barium esophagography were performed preoperatively. However, esophageal manometry and ambulatory 24-hour $\mathrm{pH}$ monitoring were not performed routinely.

The indications for the surgery were presence of symptoms (reflux or obstructive symptoms) and patients' desire for surgical repair and agreement. The latter was generally for asymptomatic cases that were incidentally discovered. Consent for surgery was obtained from patients after they had been provided with an adequate explanation of the natural course of an untreated hiatal hernia and information regarding surgery, including details of the procedure and risks involved.

Based on each patient's postoperative clinical stability, an upper gastrointestinal series using barium was performed on postoperative day 2 or 3 , and feeding was initiated after it had been confirmed that there were no abnormalities. Patients who had no symptoms with oral soft diet were discharged. All patients visited the outpatient clinic 2 weeks postoperatively for a general check-up of their state and symptoms. Follow-up visits were performed every 3 months for the first year and every 6 months thereafter.

In this study, clinical characteristics, surgery-related factors, and postoperative outcomes were analyzed for all patients.

\section{Operative techniques}

A laparoscopic approach was first attempted for all patients. The details have been described in previous MIES studies $(7,8)$. Briefly, 5 trocars with a $30^{\circ}$ angled camera and liver retractor were used. The operative procedure was similar to that used by Pierre et al. (9), and was implemented as follows (Figure 1): first, complete hernia sac dissection was performed. Then, the length of tension-free intraabdominal esophagus was measured to ensure that it was at least $2 \mathrm{~cm}$ in length; if this length was shorter than $2 \mathrm{~cm}$, modified Collis gastroplasty was performed. Subsequently, Nissen $\left(360^{\circ}\right)$ fundoplication was performed, followed by crural re-approximation. Gastroplasty was performed using end to end anastomosis (EEA) and Endo GIA linear staplers. For crural closure, only interrupted simple suture (1-0 Surgidac, Endostitch, USSC) was performed without reinforcement using mesh. Gastropexy was not performed. Surgery was converted to median laparotomy when laparoscopic surgery was deemed difficult.

\section{Statistical analysis}

All data are presented as mean \pm standard deviation or median and range, as indicated in the following parts of the text. Statistical analyses were performed using SPSS for Windows, version 19 (IBM, Chicago, IL, USA).

\section{Results}

Preoperative baseline characteristics are shown in Table 1 . The median patient age was 73 years. Nearly all patients were female $(\mathrm{n}=17,94.4 \%)$. The mean body mass index was 24.01 . Fourteen patients $(77.8 \%)$ had preoperative symptoms; 12 of these 14 had been taking proton pump inhibitors. Four patients were asymptomatic. Hiatal hernia was discovered during a routine health exam for 2 of the asymptomatic patients and through imaging studies 

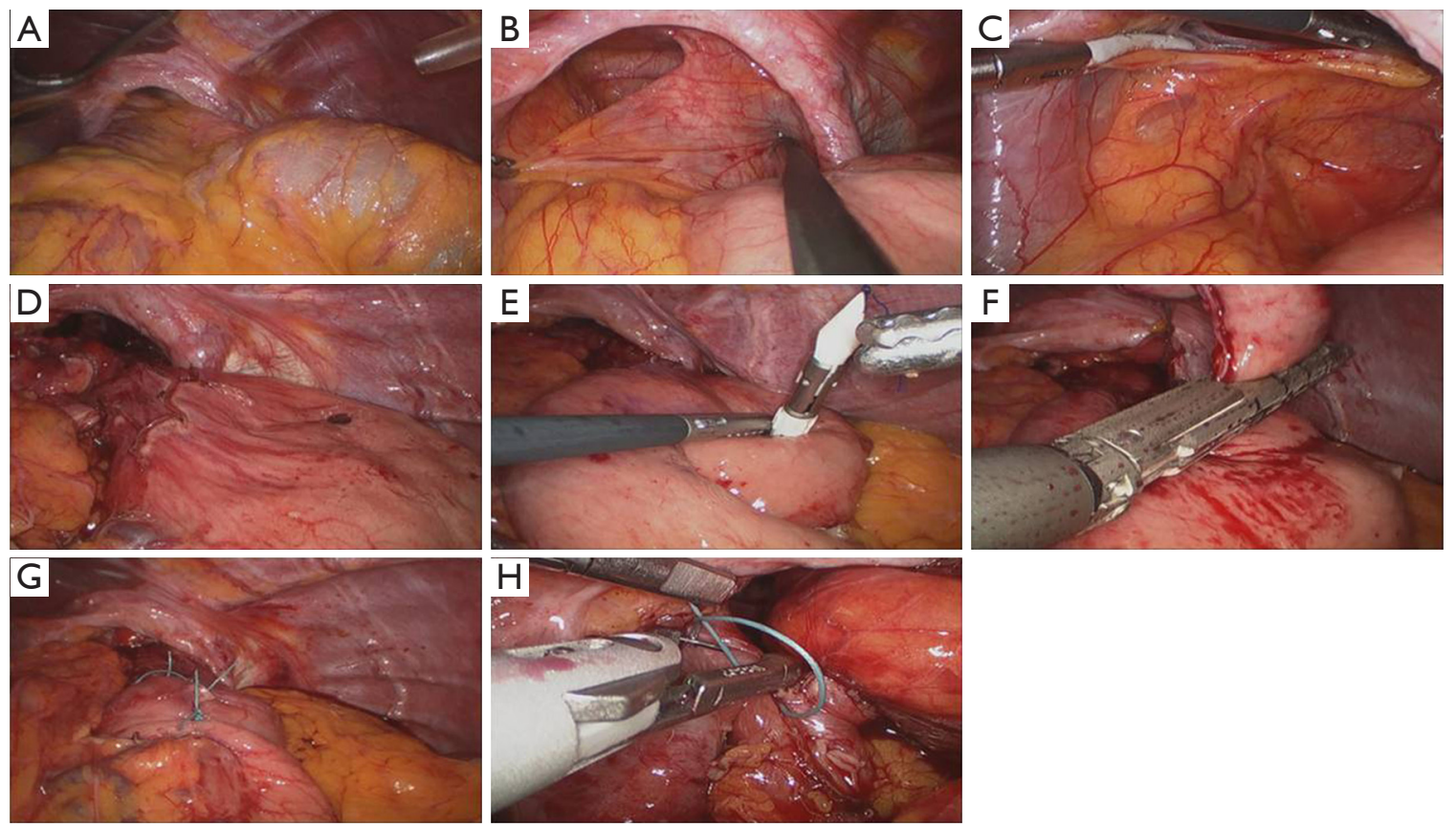

Figure 1 Intraoperative laparoscopic views with pneumoperitoneum. (A) Sliding gastroesophageal junction above the diaphragm and a herniated part of the gastric fundus (type III); (B,C) excision of the hernial sac with preservation of the crural peritoneal lining; (D) identification of inadequate length of the intra-abdominal esophagus after mediastinal esophageal mobilization; (E,F) modified Collis gastroplasty; (G) complete Nissen fundoplication; $(\mathrm{H})$ crural re-approximation.

Table 1 Preoperative baseline characteristics

\begin{tabular}{lc}
\hline Variables & Total no. of patients: 18 \\
\hline Age [years] & 73 [37-81] \\
Gender, male:female & $1: 17(94.4)$ \\
Body mass index & $24.01 \pm 4.15$ \\
Preoperative symptoms & $10(55.6)$ \\
Heart burn or epigastric pain & $4(22.2)$ \\
Dysphagia or dyspepsia & $4(22.2)$ \\
No & $12(66.7): 6$ \\
Proton pump inhibitors & \\
Yes:no & $4(22.2): 14$ \\
Prior abdominal surgery & \\
Yes:no & \\
\hline Data are $n$ (\%), median (range), and mean \pm standard deviation, \\
unless otherwise indicated.
\end{tabular}

performed to evaluate a different disease for 2 of the patients. Four patients had a history of prior abdominal surgery: two patients via laparoscopy and the other two patients via open laparotomy. At diagnosis, hiatal hernia type II was the most common (50.0\%), followed by hiatal hernia type III (27.8\%) (Table 2). Five patients underwent modified Collis gastroplasty due to inadequate length of the intra-abdominal esophagus. The mean operative time was 213.8 (range, 120-425) minutes. Two patients underwent conversion to laparotomy, one due to a relatively small abdominal cavity with large liver and the other due to severe adhesion secondary to prior surgery. The mean length of hospital stay was 6.2 (range, 4-9) days. There were no postoperative complications or deaths.

Follow-up data are shown in Table 3. The median duration of follow-up was 41 months. Three patients had symptoms at the last outpatient follow-up: reflux symptoms $(n=2)$ and dysphagia $(n=1)$. These patients showed no 
Table 2 Operative data

\begin{tabular}{lc}
\hline Variables & Total no. of patients: 18 \\
\hline Type of hiatal hernia & $3(16.7)$ \\
I & $9(50.0)$ \\
III & $5(27.8)$ \\
IV & $1(5.5)$ \\
Modified Collis gastroplasty & $5(27.8)$ \\
Operative times (minutes) & $213.8 \pm 70.1$ \\
Conversion to laparotomy & $2(11.1)$ \\
Postoperative complications & None \\
Length of hospital stay (days) & $6.2 \pm 1.5$ \\
\hline
\end{tabular}

Data are $n(\%)$, and mean \pm standard deviation, unless otherwise indicated.

notable findings on imaging examination and no difficulties with feeding; the symptoms were well-controlled with medication. One patient had radiologic recurrence 3.5 years postoperatively; after revision using open laparotomy, the patient showed improvement and is currently on follow-up.

\section{Discussion}

Manifestation of hiatal hernia can vary widely; it may be asymptomatic or may show reflux or obstructive symptoms. Although diagnosis is difficult for patients with asymptomatic hiatal hernia, the diagnosis rate has been increasing recently, in accordance with the increasing routine health examination rate $(3,10)$. Regarding GERD, which is associated with pathophysiology and symptoms, medical treatment has been the preferred approach for controlling symptoms, because of the invasiveness and efficacy of surgery (11). However, surgery-related morbidity has declined with the introduction of the laparoscopic approach; moreover, multiple studies have reported that laparoscopic surgery is superior to medical treatment in terms of long-term symptomatic improvement and costeffectiveness (12-14). Regarding hiatal hernia, some argue that watchful waiting is a reasonable alternative for the initial management of patients with asymptomatic or minimally symptomatic paraesophageal hernias (PEHs). However, most experts suggest that truly asymptomatic $\mathrm{PEHs}$ are rare. In addition, Stylopoulos et al. reported that the risk of progression from asymptomatic to symptomatic
Table 3 Follow-up data

\begin{tabular}{lc}
\hline Variables & Total no. of patients: 18 \\
\hline Duration of follow-up [months] & 41 [10-107] \\
Current symptoms & $3(16.7)$ \\
Yes & 15 \\
No & $1(5.5)$ \\
Recurrence &
\end{tabular}

Data are $\mathrm{n}(\%)$, median (range), unless otherwise indicated.

PEH is approximately $14 \%$ per year (15). We provided a detailed explanation of these considerations to patients, prior to obtaining their consent to undergo surgery.

Laparoscopic repair of $\mathrm{PEH}$ has become the preferred surgical approach because it reduces pain, morbidity, and the length of hospital stay, compared to the conventional open approach $(16,17)$; however, it has also been reported to have a relatively high recurrence rate (18). Recently, the recurrence rate may now be lowered while maintaining the above benefits, based on the evolution of the laparoscopic technique and accumulation of experience (19).

Although many studies, including randomized controlled trials, have been conducted to determine whether to perform reinforcement using prosthetic patch, lengthening procedure for short esophagus, gastropexy, or fundoplication (including type of fundoplication) in order to increase treatment efficacy while maintaining a low recurrence rate, such approaches remain controversial (20-25). The Society of American Gastrointestinal and Endoscopic Surgeons (SAGES) summarized the technical considerations for surgery in their guidelines for the management of hiatal hernia in 2013 (26). Notably, these guidelines require appropriate interpretation depending on the quality of the available evidence; however, such recommendations, combined with expert opinion, could be reliable and useful. One of the important technical considerations suggested with strong evidences is that the gastroesophageal junction should be placed in the infradiaphragmatic position. O'Rourke et al. suggested that a short esophagus can be resolved through extended mediastinal dissection, thereby replacing Collis gastroplasty (27). However, in some of our cases, the length of intra-abdominal esophagus remained short despite sufficient mediastinal dissection; in such cases, gastroplasty was performed without hesitation $(27.8 \%$ of all patients).

Although divisions of surgery departments can vary 
among countries, hiatal hernia repair is generally performed within departments of general and/or digestive (visceral) surgery. Unlike some western countries, in Korea, the department of surgery is not divided depending on disease or organ; thus, thoracic and abdominal surgery are clearly separated. In such systems, it may be unclear which surgical department should perform this surgery. Although MIES using thoracoscopy and laparoscopy is widely performed on patients with esophageal cancer, it remains uncommon for thoracic surgeons to perform laparoscopic surgeries in many countries. However, thoracic surgeons who have experience with laparoscopic surgery through MIES are adequately capable of performing this surgery without considerable difficulty. Indeed, the complexity of hiatal hernia surgery requires a significant learning curve. Okrainec et al. reported that surgeons need at least 20 cases of experience in order to achieve an appropriately low recurrence rate (28). We were able to successfully perform this surgery without complications and with a low recurrence rate because we began performing this surgery after accumulating sufficient experience in laparoscopic surgery with MIES, and followed the SAGES guidelines.

Limitations of our retrospective study included its small sample size and the relatively long period of time involved. However, the incidence and diagnosis rate of hiatal hernia is not high in eastern countries including Korea. For this reason, there have been few reports regarding surgical treatment of the disease; thus, we aimed to share our surgical experience.

We conclude that laparoscopic repair of hiatal hernia is a feasible technique with satisfactory surgical outcomes. Although it is a complex operation with a substantial learning curve, thoracic surgeons who have adequate experience with laparoscopy would be capable of performing the operation.

\section{Acknowledgments}

Funding: This study was supported by a grant (HCRI14020-1) Chonnam National University Hwasun Hospital Institute for Biomedical Science.

\section{Footnote}

Conflicts of Interest: The authors have no conflicts of interest to declare.

Ethical Statement: The authors are accountable for all aspects of the work in ensuring that questions related to the accuracy or integrity of any part of the work are appropriately investigated and resolved. The present study protocol was reviewed and approved by the Institutional Review Board of Chonnam National University Hwasun Hospital, which waived the requirement for informed patient consent based on the retrospective nature of the work (approval No. CNUHH-2019-056).

\section{References}

1. Petersen H, Johannessen T, Sandvik AK, et al. Relationship between endoscopic hiatus hernia and gastroesophageal reflux symptoms. Scand J Gastroenterol 1991;26:921-6.

2. El-Serag HB, Sweet $\mathrm{S}$, Winchester CC, et al. Update on the epidemiology of gastro-oesophageal reflux disease: a systematic review. Gut 2014;63:871-80.

3. Kim KM, Cho YK, Bae SJ, et al. Prevalence of gastroesophageal reflux disease in Korea and associated health-care utilization: a national population-based study. J Gastroenterol Hepatol 2012;27:741-5.

4. Schlottmann F, Herbella FA, Allaix ME, et al. Surgical treatment of gastroesophageal reflux disease. World J Surg 2017;41:1685-90.

5. Siegal SR, Dolan JP, Hunter JG. Modern diagnosis and treatment of hiatal hernias. Langenbecks Arch Surg 2017;402:1145-51.

6. Luketich JD, Nason KS, Christie NA, et al. Outcomes after a decade of laparoscopic giant paraesophageal hernia repair. J Thorac Cardiovasc Surg 2010;139:395-404.e1.

7. Song SY, Na KJ, Oh SG, et al. Learning curves of minimally invasive esophageal cancer surgery. Eur J Cardiothorac Surg 2009;35:689-93.

8. Yun JS, Na KJ, Song SY, et al. Comparison of perioperative outcomes following hybrid minimally invasive versus open Ivor Lewis esophagectomy for esophageal cancer. J Thorac Dis 2017;9:3097-104.

9. Pierre AF, Luketich JD, Fernando HC, et al. Results of laparoscopic repair of giant paraesophageal hernias: 200 consecutive patients. Ann Thorac Surg 2002;74:1909-15; discussion 1915-6.

10. Katz PO, Gerson LB, Vela MF. Guidelines for the diagnosis and management of gastroesophageal reflux disease. Am J Gastroenterol 2013;108:308-28; quiz 329.

11. Iwakiri K, Kinoshita Y, Habu Y, et al. Evidence-based clinical practice guidelines for gastroesophageal reflux disease 2015. J Gastroenterol 2016;51:751-67.

12. Mehta S, Bennett J, Mahon D, et al. Prospective trial of 
laparoscopic nissen fundoplication versus proton pump inhibitor therapy for gastroesophageal reflux disease: seven-year follow-up. J Gastrointest Surg 2006;10:1312-6; discussion 1316-7.

13. Anvari M, Allen C, Marshall J, et al. A randomized controlled trial of laparoscopic Nissen fundoplication versus proton pump inhibitors for the treatment of patients with chronic gastroesophageal reflux disease (GERD): 3-year outcomes. Surg Endosc 2011;25:2547-54.

14. Grant AM, Boachie C, Cotton SC, et al. Clinical and economic evaluation of laparoscopic surgery compared with medical management for gastro-oesophageal reflux disease: 5 -year follow-up of multicentre randomised trial (the REFLUX trial). Health Technol Assess 2013;17:1-167.

15. Stylopoulos N, Gazelle GS, Rattner DW. Paraesophageal hernias: operation or observation? Ann Surg 2002;236:492500; discussion 500-1.

16. Schauer PR, Ikramuddin S, McLaughlin RH, et al. Comparison of laparoscopic versus open repair of paraesophageal hernia. Am J Surg 1998;176:659-65.

17. Karmali S, McFadden S, Mitchell P, et al. Primary laparoscopic and open repair of paraesophageal hernias: a comparison of short-term outcomes. Dis Esophagus 2008;21:63-8.

18. Dallemagne B, Kohnen L, Perretta S, et al. Laparoscopic repair of paraesophageal hernia. Long-term follow-up reveals good clinical outcome despite high radiological recurrence rate. Ann Surg 2011;253:291-6.

19. Zehetner J, Demeester SR, Ayazi S, et al. Laparoscopic versus open repair of paraesophageal hernia: the second decade. J Am Coll Surg 2011;212:813-20.

20. Oelschlager BK, Pellegrini CA, Hunter J, et al. Biologic

Cite this article as: Yun JS, Na KJ, Song SY, Kim S, Kim E, Jeong IS, Oh SG. Laparoscopic repair of hiatal hernia. J Thorac Dis 2019;11(9):3903-3908. doi: 10.21037/jtd.2019.08.94 prosthesis reduces recurrence after laparoscopic paraesophageal hernia repair: a multicenter, prospective, randomized trial. Ann Surg 2006;244:481-90.

21. Furnée E, Hazebroek E. Mesh in laparoscopic large hiatal hernia repair: a systematic review of the literature. Surg Endosc 2013;27:3998-4008.

22. Lin E, Swafford V, Chadalavada R, et al. Disparity between symptomatic and physiologic outcomes following esophageal lengthening procedures for antireflux surgery. J Gastrointest Surg 2004;8:31-9; discussion 38-9.

23. Du X, Wu JM, Hu ZW, et al. Laparoscopic Nissen (total) versus anterior $180^{\circ}$ fundoplication for gastro-esophageal reflux disease: a meta-analysis and systematic review. Medicine (Baltimore) 2017;96:e8085.

24. Morris-Stiff G, Hassn A. Laparoscopic paraoesophageal hernia repair: fundoplication is not usually indicated. Hernia 2008;12:299-302.

25. Ponsky J, Rosen M, Fanning A, et al. Anterior gastropexy may reduce the recurrence rate after laparoscopic paraesophageal hernia repair. Surg Endosc 2003;17:1036-41.

26. Kohn GP, Price RR, DeMeester SR, et al. Guidelines for the management of hiatal hernia. Surg Endosc 2013;27:4409-28.

27. O'Rourke RW, Khajanchee YS, Urbach DR, et al. Extended transmediastinal dissection: an alternative to gastroplasty for short esophagus. Arch Surg 2003;138:735-40.

28. Okrainec A, Ferri LE, Feldman LS, et al. Defining the learning curve in laparoscopic paraesophageal hernia repair: a CUSUM analysis. Surg Endosc 2011;25:1083-7. 